\title{
Neues von der Tariffront
}

\section{Urs Saxer}

Prof. Dr. iur., LL.M (Columbia Law School N.Y.), Rechtsanwalt

\author{
Die geltende Tarifstruktur TARMED hat ausgedient. Nachdem mit dem Kranken- \\ kassenverband santésuisse kein Konsens gefunden werden konnte, erarbeiteten \\ die FMH, der Krankenkassenverband curafutura und die Medizinaltarifkommis- \\ sion (MTK) mit TARDOC eine neue, zeitgemässe Tarifstruktur. Doch bis TARDOC in \\ Kraft treten kann, sind noch einige Hürden zu nehmen. Santesuisse wird wohl \\ nicht mitmachen. Wird TARDOC, vielleicht modifiziert, durch den Bundesrat \\ festgesetzt?
}

Der TARMED wartet schon sein Längerem auf seine Ablösung. Noch immer ist er als seit 15 Jahren geltender, gesamtschweizerischer Einzelleistungstarif im ambulanten Bereich zentrale Grundlage für die Berechnung der Vergütung der ärztlichen Leistungserbringer. Aber alle wissen es: Der TARMED hat ausgedient. Er ist ein Modell, dessen Verfallsdatum bereits vor einigen Jahren abgelaufen ist: Von den Tarifpartnern nicht mehr gepflegt und durch zwei bundesrätliche Eingriffe zusätzlich verunstaltet, hat er sich von einem betriebswirtschaftlichen Hintergrund immer

Alle wissen es: Der Tarmed hat ausgedient. Er ist ein Modell, dessen Verfallsdatum bereits vor einigen Jahren abgelaufen ist.

mehr gelöst und siecht, teilweise falsche, teilweise ungerechte Anreize setzend, vor sich hin. Er basiert auf überholten Daten, z.B. mit Bezug auf das ärztliche Referenzeinkommen, und reflektiert den technischen Fortschritt zu wenig. Das Verhältnis der verschiedenen Leistungen untereinander sowie zwischen ärztlichen und technischen Leistungen muss wieder ins Lot gebracht, die medizinische und die betriebswirtschaftliche Welt stärker verbunden werden. Er sollte möglichst korrekt die Realitäten bei der Erbringung ambulanter Leistungen reflektieren, damit diese sachgerecht und betriebswirtschaftlich korrekt berechnet und damit auch entgolten werden können.

\section{Einvernehmliche Revision gescheitert}

Alle wissen dies eigentlich, und schon seit Jahren gibt es Bemühungen zwischen den Tarifpartnern, die geltende Tarifstruktur abzulösen. Die Totalblockade von santésuisse hat dies verhindert. Diese ist dem ursprünglich einzigen Krankenkassenverband nicht gut bekommen. Er vertritt zwar immer noch knapp mehr als die Hälfte der Versicherer, aber eben nicht wie früher alle. Mit dem Verlust des Vertretungsmonopols geht auch ein Legitimitätsverlust einher. Was er aber immer noch voll ausspielen kann, ist ein Stör- und Blockierpotential, und dies setzt er mit grosser Konsequenz um. Der Verband machte bei der TARMED Suisse AG nicht mit, in deren Rahmen die Tarifstruktur hätte einvernehmlich revidiert werden sollen. Dieses Projekt scheiterte bekanntlich 2016 in der FMH-Urabstimmung. Er machte auch nicht mit in der ats-tms AG, der Nachfolgeorganisation zur Revision des TARMED. Mit Getöse schied dort auch, aus nicht völlig klaren Gründen, $\mathrm{H}+$ aus. All dies zeigt, dass es sehr schwierig ist, unter den Tarifparteien einen Konsens über einen neuen Einzelleistungstarif zu finden. Es werden, auf dem Verhandlungsweg, nie alle an Bord sein. Trotzdem, oder vielleicht gerade deswegen, konnten die verbleibenden Tarifpartner, also die FMH, Curafutura und

Der Bundesrat hat allerdings die Möglichkeit, die Struktur festzusetzen, also zu verordnen.

die MTK, im kleineren Kreis mit TARDOC eine neue Tarifstruktur schaffen. Diese soll im Rahmen der ats-tms AG kontinuierlich gepflegt und weiterentwickelt werden. Zuerst muss sie allerdings die bisherige Struktur überhaupt ablösen können. In der FMH hat sich die Ärztekammer mit einer überzeugenden Mehrheit hinter das Projekt gestellt. In der Zwischenzeit haben die daran beteiligten Tarifpartner TARDOC 
dem Bundesrat zur Genehmigung zugestellt. Aber eben: ohne santésuisse und $\mathrm{H}+$. Letztere schliesst eine nachträgliche Beteiligung allerdings nicht grundsätzlich aus.

\section{Mögliche Umgehung der Blockadepolitik}

Die Inkraftsetzung des TARDOC ist mit etlichen Problemen verbunden. Da Einzelleistungstarife gemäss KVG gesamtschweizerisch Geltung haben müssen, verlangt der Bundesrat für eine Genehmigung, dass hinter einer neuen Struktur die Mehrheit der betroffenen Branchen steht. Dies ist im Moment bei den Versicherern wegen des Abseitsstehens von santésuisse nicht der Fall. Der Bundesrat hat allerdings die Möglichkeit, die Struktur festzusetzen, also zu verordnen. Dies kann er, ohne die Struktur zu verändern, oder, was die wahrscheinlichere Variante ist, er kann sie modifizieren. Mit der Festsetzung hätte dann der Tarif gesamtschweizerische Geltung und würde den TARMED ablösen. Dies ist eine Möglichkeit, die Blockadepolitik von santésuisse zu umgehen. Bevor der neue Tarif verordnet wird, wird wohl ein Vernehmlassungsverfahren durchgeführt werden, in dessen Rahmen sich unter anderem auch santésuisse und H+ äussern können. Die Resultate der Vernehm-

\section{Das Wichtigste in Kürze}

- Die FMH, Curafutura und die MTK haben im kleineren Kreis mit TARDOC eine neue Tarifstruktur geschaffen, welche den ausgedienten TARMED ersetzen könnte.

- Für eine Genehmigung des TARDOC durch den Bundesrat bräuchte es grundsätzlich eine Mehrheit der betroffenen Branchen, was aufgrund des Abseitsstehens von santésuisse nicht der Fall ist. Der Bundesrat hat jedoch die Möglichkeit, die neueTarifstruktur zu verordnen.

- Über die Auswirkungen einer Einführung von TARDOC au den Rahmenvertrag TARMED sind die Meinungen geteilt. Verschiedene Szenarien sind denkbar.

\section{L'essentiel en bref}

- La FMH, Curafutura et la CTM ont créé en cercle restreint le TARDOC, une nouvelle structure tarifaire susceptible de remplacer leTARMED, qui a fait son temps.

- Pour que le TARDOC soit approuvé par le Conseil fédéral, la majorité des branches concernées est en principe requise, or ce n'est pas le cas ici du fait de la non-participation de santésuisse. Le Conseil fédéral a toutefois la possibilité de décréter la nouvelle structure tarifaire.

- Les avis au sujet des répercussions de l'introduction de TARDOC sur la convention-cadreTARMED sont partagés. Différents scénarios sont envisageables. lassung können dann in den festgesetzten Tarif einfliessen. Entscheidend wird für die Frage der Festsetzung unter anderem sein, ob in den Augen der Regierung die Struktur in der Sache überzeugt. Hinzu kommen politische Überlegungen.

\section{Kostenneutralität als Grundbedingung}

Ein zentrales Problem ist die Frage der Kostenneutralität. Diese kommt gesetzlich bei einem Wechsel des Tarifsystems zum Zug. TARDOC ist wie der TARMED ein Einzelleistungstarif, beruht also nicht auf einem

Der neue Tarif ist indes ohne Kostenneutralität nicht zu haben. Die Frage ist, wie sie umgesetzt wird.

neuen System. Trotzdem verlangt der Bundesrat die Einhaltung der Kostenneutralität mit dem Argument, dass die Unterschiede zum TARMED derart gross seien bzw. sein müssen, dass die Auswirkungen einem Systemwechsel gleichkämen. Gerade die Einhaltung der Kostenneutralität mittels einer Normierung der Struktur wurde aber in der FMH-Urabstimmung von 2016 wuchtig abgelehnt. Der neue Tarif ist indes ohne Kostenneutralität nicht zu haben. Die Frage ist, wie sie umgesetzt wird. Die Ärzteschaft hat hierzu ein Konzept vorgelegt, das einen Korridor vorsieht und in einer Einführungsphase regelmässige Anpassungen des Tarifs dort zulässt, wo sich in der Anwendung dysfunktionale Auswirkungen zeigen. Es wird sich zeigen, ob dieses ähnlich schon bei der Einführung des TARMED verwendete Konzept die Gnade der Regierung findet.

\section{Verschiedene Zukunftsszenarien}

Was geschieht mit dem Rahmenvertrag TARMED, wenn TARDOC in Kraft gesetzt wird? Die Meinungen hierüber sind geteilt. santésuisse ist der Auffassung, bereits mit der Einreichung des TARDOC-Genehmigungsgesuchs müsse der Rahmenvertrag gekündigt werden. Dafür spricht, dass der Rahmenvertrag sich ausdrücklich auf den TARMED bezieht. Wenn allerdings ein Einzelleistungstarif nur revidiert und auf dem Weg einer Festsetzung durch einen anderen abgelöst wird, hat dies nicht zwingend die Auflösung des Rahmenvertrags zur Konsequenz. Dieser enthält denn auch diverse Bestimmungen, die ohne Probleme weitergelten können, z.B. die Regelungen zur Dignität, zur Rechnungstellung, zu den Beitrittsgebühren, sodann Regelungen zur EAN-Nummer/Registriernummer, zur Anstellung von Ärzten und zur Leistungserbringung 
Prof. Dr. iur., LL.M. Urs Saxer Steinbrüchel Hüssy Rechtsanwälte Grossmünsterplatz 8 CH-8001 Zürich Saxer[at]steinlex.ch im Rahmen von juristischen Personen, zu den Vertrauensärzten und zu den paritätischen Vertrauenskommissionen. Nicht zu vergessen ist, dass mit einer Kündigung oder Aufhebung des Rahmenvertrags auch die kantonalen Anschlussverträge dahinfallen würden und damit auch die in Anhang B zu den Anschlussverträgen vereinbarten Taxpunktwerte. Eine Kündigung oder ersatzlose Aufhebung des Rahmenvertrags muss angesichts der möglichen Konsequenzen damit wohl überlegt sein. Allerdings gibt es durchaus offene Fragen mit Bezug auf eine allfällige Weitergeltung des Vertrags. So wurde dieser mit einer an der Entwicklung der neuen Struktur nicht beteiligten Gruppierung geschlossen, nämlich santésuisse, und sein Gegenstück im Spitalbereich ist seit 2017 gekündigt. Offen ist auch, wie Curafutura zur (Weiter-)Geltung des Rahmenvertrags steht. Angesichts dessen erscheint ein pragmatisches Vorgehen wohl am besten. Nicht verhindert werden kann allerdings eine Kündigung durch santésuisse. Es gibt also diverse mögliche Szenarien.

Ein weiterer Punkt sind die kantonalen Regelungen der Taxpunktwerte (TPW). Die Struktur ist ja eine $\mathrm{Zu}$ ständigkeit des Bundes, die Festlegung des TPW demgegenüber eine kantonale Kompetenz. Was ist, was wäre der Einfluss einer kostenneutralen Einführung des TARDOC auf die kantonale TPW-Ebene? Grundsätzlich sind die Ebenen verschieden: Gründe für eine Erhöhung oder Senkung des Taxpunktwertes sind strukturunabhängig. Eine Vermischung wäre daher unzulässig: Es darf nicht erhöht oder gesenkt werden, um wirtschaftliche Auswirkungen der Strukturrevision auf kantonaler Ebene zu kompensieren. Es ist aber denkbar, dass aus anderen Gründen sich in einzelnen Kantonen eine Erhöhung oder Senkung aufdrängt. Indes, ein faktisches Moratorium in einer Einführungsphase kann wohl nicht ausgeschlossen werden, um die Auswirkungen der neuen Struktur unbeeinflusst von weiteren Faktoren zu analysieren und allenfalls erforderliche Tarifstrukturanpassungen vorzunehmen. Für laufende Festsetzungsverfahren und beantragte Genehmigungen kann dies eine Sistierung bedeuten.

\section{Die Zukunft ist ungewiss}

Dies alles zeigt: Die Ablösung des TARMED ist ein komplexer Vorgang. Erschwerend kommt hinzu, dass das KVG konzeptionell stark auf den Konsens der Tarifpartner setzt, also auf vertragliche Regelungen, es aber immer schwieriger wird, diesen Konsens zu finden. Im Bereich der Revision der Tarifstruktur ist dies als Folge der intransigenten Haltung von santésuisse schlicht unmöglich geworden. Es kann sein, dass der politisch gut vernetzte und nach wie vor grösste Kassenverband darauf spekuliert, die Versicherer würden mit weiteren Tarifeingriffen oder einem festgesetzten neuen Einzelleistungstarif besser fahren als mit einer vertraglichen Einigung. Es ist zu hoffen, dass dieser Kalkül nicht aufgeht. Das völlige Abseitsstehen bei der Erarbeitung eines neuen Einzelleistungstarifs dürfte santésuisse auf jeden Fall bis jetzt eher geschadet haben.

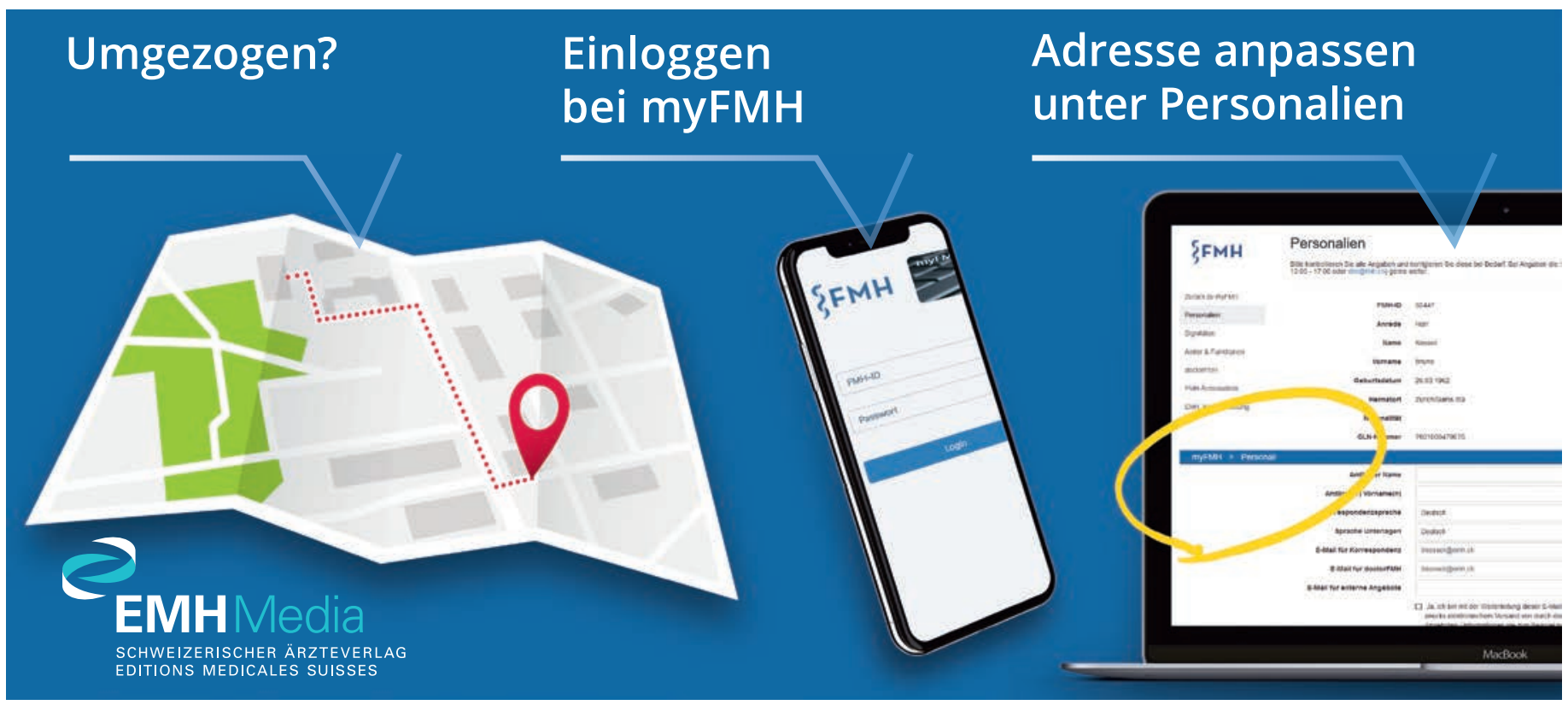

